\title{
Measurement of the inclusive muon differential cross section with the ATLAS detector at LHC
}

\section{Biagio Di Micco*}

European Organization for Nuclear Research (CERN)

E-mail: biagio.di.micco@cern.ch

\begin{abstract}
We present the measurement of the inclusive muon differential cross section $\left(\mathrm{d} \sigma / \mathrm{dp}_{T}\right)$ in $p p$ collisions at $\sqrt{s}=7 \mathrm{TeV}$ with the ATLAS detector. The analysis is performed in the pseudorapidity interval $|\eta|<2.5$ for muons of transverse momentum $4<\mathrm{p}_{T}<100 \mathrm{GeV}$ and with an integrated luminosity of $1.4 \mathrm{pb}^{-1}$. The result is compared with a Next-To-Leading Order prediction for the heavy flavour production with Next to Leading Log high $\mathrm{p}_{T}$ resummation and with an MC@NLO prediction for $\mathrm{W}$ and $\mathrm{Z}$ bosons production. The measurement is sensitive for the first time to the Next to Leading Log contribution to heavy flavour production in hadronic interactions.
\end{abstract}

The 2011 Europhysics Conference on High Energy Physics, EPS-HEP 2011,

July 21-27, 2011

Grenoble, Rhône-Alpes, France

${ }^{*}$ Speaker. 


\section{Introduction}

The spectrum of muons produced close to the interaction point in $p p$ interactions is sensitive to heavy flavour production in the range $4<\mathrm{p}_{T}<100 \mathrm{GeV}$. For $p_{T}>30 \mathrm{GeV}$ the W/Z contribution becomes important and is subtracted in order to measure the heavy flavour component. A measurement in this region is useful to optimise theoretical computations and Monte Carlo generators to improve the modeling of the heavy flavour production mechanism.

Nowadays theoretical computations from pQCD [1] are available allowing predictions of the muon spectrum from the QCD lagrangian and data-driven non perturbative corrections, dependent upon fragmentation function extracted from LEP data and heavy flavours decays and form factors extracted from B-factories. These calculations have been performed at Next to Leading Order (NLO) + Next to Leasing Log (NLL). No experiment up to now was sensitive to the NLL contribution. At high $\mathrm{p}_{T}$ the NLO+NLL prediction becomes more accurate due to the reduction of the scale uncertainty and the deviation between the NLO computation and the NLO+NLL becomes larger, allowing to disentangle the NLL contribution. The results presented here have been also published in [2].

\section{The measurement}

The measured differential cross-section within the kinematic acceptance of the charged lepton is extracted using

$$
\frac{d \sigma}{d p_{\mathrm{T}}} \approx \frac{\Delta \sigma_{i}}{\Delta p_{\mathrm{T}_{i}}}=\frac{N_{\mathrm{sig}_{i}}}{\Gamma_{\mathrm{bin}_{i}} \cdot \int \mathscr{L} d t} \cdot \frac{C_{\text {migration }_{i}}}{\varepsilon_{\left(\text {(reco }_{\mathrm{PID})}\right.} \cdot \varepsilon_{\text {trigger }_{i}}},
$$

where $N_{\mathrm{sig}_{i}}$ is the number of muons with reconstructed $p_{\mathrm{T}}$ in bin $i$ of width $\Gamma_{\mathrm{bin}_{i}}, \int \mathscr{L} d t$ is the integrated luminosity, $\varepsilon_{\text {trigger }_{i}}$ is the trigger efficiency and $\varepsilon_{(\text {reco }+ \text { PID })_{i}}$ is the combined reconstruction and identification efficiency. $C_{\text {migration }_{i}}$ is the bin migration correction factor, defined as the ratio of the number of muons of true $p_{\mathrm{T}}$ in bin $i$ to the number reconstructed in the same $p_{\mathrm{T}}$ bin. The analysis is based on a data sample collected at $\sqrt{s}=7 \mathrm{TeV}$ in 2010 corresponding to an integrated luminosity of $1.42 \pm 0.05 \mathrm{pb}^{-1}$. The difference in $p_{\mathrm{T}}$ between the Inner Detector (ID) and the Muon Spectrometer (MS) tracking system, $\Delta p_{\mathrm{T}}=p_{\mathrm{T}}^{\mathrm{ID}}-p_{\mathrm{T}}^{\mathrm{MS}}$, where both momenta are extrapolated to the interaction point, is sensitive to the origin of the muons: signal, $\pi / K$ decays and fakes. A fit to the data distribution is performed to extract the signal component $\left(N_{\mathrm{sig}_{i}}\right)$ using templates from simulated data samples generated in order to estimate background and to correct for the trigger efficiency, the reconstruction efficiency and the detector resolution.

\subsection{Inclusive muon differential cross section}

The signal yield $\left(N_{\mathrm{sig}_{i}}\right)$ has been corrected for the trigger and reconstruction efficiencies and unfolded from the detector response. Figure 1, left shows the resulting inclusive muon differential cross-section for muons within $|\eta|<2.5$ as a function of $p_{\mathrm{T}}$, compared to the overall theoretical expectation. The systematic uncertainty ranges from $5 \%$ to $8 \%$ and is dominated by the uncertainty on the signal fraction extraction. The expected $W / Z$ component comes from MC@NLO using the 

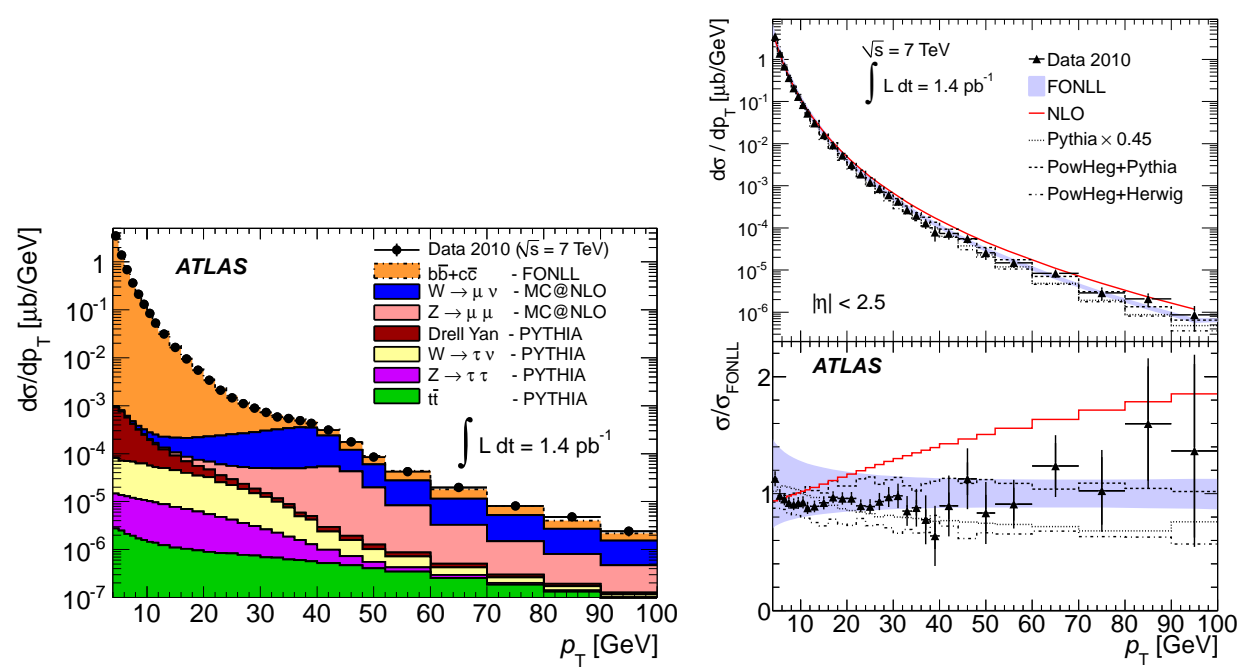

Figure 1: (Left) Muon differential cross-section as a function of the muon transverse momentum for $|\eta|<$ 2.5 compared to theoretical predictions from each contributions. (Right) Muon differential cross section as a function of $p_{\mathrm{T}}$ after subtraction of the $\mathrm{W} / \mathrm{Z} / \gamma^{(*)}$ contribution.

CTEQ 6 . 6 PDFs, normalised to the cross-sections for muons measured by ATLAS [3]. The FONLL prediction is used for the heavy-flavour component and the remaining, small contributions, are obtained from PYTHIA simulation. The theoretical uncertainty is dominated by the heavy-flavour prediction, being approximately $20 \%$, and is not shown in the figure.

In Fig. 1, right we show the muon cross-section after subtracting all contributions except the heavy flavours over the full $p_{\mathrm{T}}(4-100 \mathrm{GeV})$ and pseudorapidity $(|\eta|<2.5)$ ranges. The measured heavy-flavour cross-section is compared to the FONLL calculations. The theoretical uncertainties (shown as a band in the figure) are around $20-40 \%$, decreasing with $p_{\mathrm{T}}$. The results are also compared to the NLO predictions of the POWHEG program, interfaced to either PYTHIA or HERWIG for the parton shower simulation, and to the LO plus parton shower predictions of PYTHIA. Comparisons are also made to the NLO central value expectation obtained from the FONLL program by excluding the NLL resummation part of the perturbative QCD calculation. The data deviate significantly from the NLO prediction, showing sensitivity to the NLL resummation term in the pQCD calculation for the first time in heavy-flavor production at hadron colliders.

\section{References}

[1] M. Cacciari, S. Frixione, M. L. Mangano, P. Nason and G. Ridolfi, JHEP 0407 (2004) 033.

[2] Measurements of the electron and muon inclusive cross-sections in proton-proton collisions at $\sqrt{s}=7$ TeV with the ATLAS detector, ATLAS Collaboration, arXiv:1109.0525 [hep-ex] (2011) - Submitted to Phys. Lett. B.

[3] ATLAS Collaboration, ATLAS-CONF-2011-046 (2011). 